\title{
Iron-catalyzed decarboxylative alkenylation of cycloalkanes with arylvinyl carboxylic acids via a radical process
}

\author{
Jincan Zhao ${ }^{1}$, Hong Fang ${ }^{1}$, Jianlin $\operatorname{Han}^{* 1,2}$ and Yi Pan*1
}

\section{Full Research Paper}

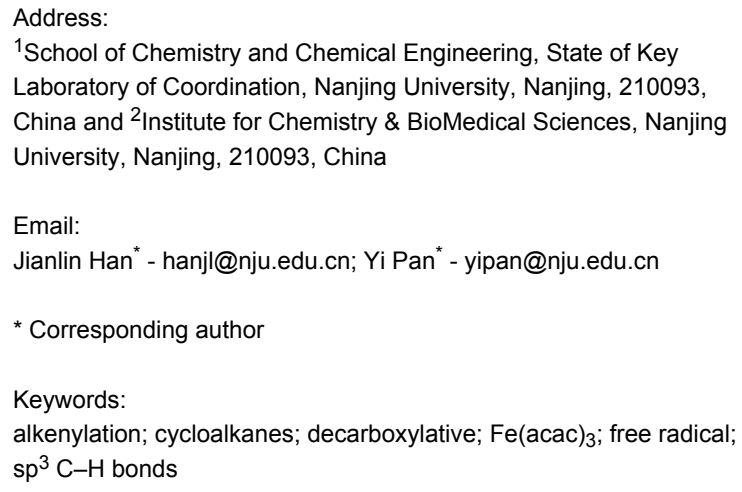

\author{
Beilstein J. Org. Chem. 2013, 9, 1718-1723. \\ doi:10.3762/bjoc.9.197 \\ Received: 01 July 2013 \\ Accepted: 02 August 2013 \\ Published: 21 August 2013 \\ This article is part of the Thematic Series "Organic free radical chemistry". \\ Guest Editor: C. Stephenson \\ (C) 2013 Zhao et al; licensee Beilstein-Institut. \\ License and terms: see end of document.
}

\begin{abstract}
A Fe(acac) $)_{3}$-catalyzed decarboxylative coupling of 2-(aryl)vinyl carboxylic acids with cycloalkanes was developed by using DTBP as an oxidant through a radical process. This reaction tolerates a wide range of substrates, and products are obtained in good to excellent yields (71-95\%). The reaction also shows excellent stereoselectivity, and only trans-isomers are obtained.
\end{abstract}

\section{Introduction}

Direct $\mathrm{C}-\mathrm{H}$ functionalization has become one of the most useful and attractive tools in organic chemistry because it can construct carbon-carbon or carbon-heteroatom bonds in a highly atom economical manner [1-8]. Among all these $\mathrm{C}-\mathrm{H}$ functionalization methods, the direct $\mathrm{C}\left(\mathrm{sp}^{3}\right)-\mathrm{H}$ functionalization attracts particular attention due to its low reactivity and challenging activation [9-11]. In previous studies, numerous transition-metal-catalyzed processes, such as $\mathrm{Pd}$ [12-18], $\mathrm{Cu}$ [19-23], Ru [24-27], Rh [28-31], Co [32-34], Au [35,36], Ir [3739], Fe [40-43] and other metals [44-47], have been developed for $\mathrm{sp}^{3} \mathrm{C}-\mathrm{H}$ activation reactions. Additionally, metal-free methodologies, which use TBHP, $\mathrm{PhI}(\mathrm{OAc})_{2}, \mathrm{TBAI}, \mathrm{I}_{2}$ or
Lewis/Brønsted acids, have also been employed for cross-dehydrogenative coupling reactions [48-57].

Owing to the general low reactivity of cycloalkane $\mathrm{C}\left(\mathrm{sp}^{3}\right)-\mathrm{H}$ bonds, the direct alkenylation of cycloalkanes with high selectivity and stereospecificity remains a great challenge and attracted a lot of attention in the past years. In 1996, the Fuchs group described the alkenylation of cyclohexane by a radical reaction with vinyl triflone [58]. In 2003, the Yao group reported that styryl cycloalkanes were prepared based on a radical substitution of cyclohydrocarbon units to $(E)-\beta$ nitrostyrenes by using the radical initiator benzoyl peroxide 
[59]. Recently, the Liu group developed a copper-catalyzed decarboxylative coupling of vinylic carboxylic acids with simple alcohols and ethers in high yields. Cycloalkanes were also investigated in this catalytic system, though only moderate yields were obtained [60].

Several other groups have also found that $\mathrm{Pd}, \mathrm{Ag}$ or $\mathrm{Cu}$ could catalyze the decarboxylative coupling of various aromatic, alkenyl, and alkynyl carboxylic acids [61-68]. Our group was surprised to find that low-cost $\mathrm{Fe}(\mathrm{acac})_{3}$ could catalyze the direct alkenylation of cyclohexane $\mathrm{sp}^{3} \mathrm{C}-\mathrm{H}$ bonds by decarboxylative couplings with high efficiency.

\section{Results and Discussion}

We initiated our investigation by reacting cinnamic acid (1a, $0.3 \mathbf{m m o l})$ with cyclohexane $(\mathbf{2 a}, 2 \mathrm{~mL})$ in the presence of iron(II) chloride tetrahydrate $(20 \mathrm{~mol} \%)$ and 2.0 equiv of di-tert-butyl peroxide (DTBP) as the oxidant at $120{ }^{\circ} \mathrm{C}$ under nitrogen, which provided the expected $(E)-(2-$ cyclohexylvinyl)benzene (3a), but in a moderate $54 \%$ yield (Table 1, entry 1). The use of aqueous TBHP as oxidant instead of DTBP reduced the yield to only $38 \%$ (Table 1 , entry 2 ). With the help of 1,10-phenanthroline (30 mol \%) as the ligand, the yield could be slightly improved to $68 \%$ (Table 1 , entry 3 ). Iron(III) acetylacetonate provided a superior yield (91\%) compared to the other Fe salts such as $\mathrm{FeCl}_{3}$, ferrocene, $\mathrm{Fe}_{2} \mathrm{O}_{3}$ and $\mathrm{Fe}_{3} \mathrm{O}_{4}$ tested (Table 1, entries 4-8). Application of other oxidants such as $\mathrm{K}_{2} \mathrm{~S}_{2} \mathrm{O}_{8}, \mathrm{H}_{2} \mathrm{O}_{2}$ (30\% aqueous solution) or TBPB did not afford any improvements (Table 1, entries 9-11). A decreased loading of $\mathrm{Fe}(\mathrm{acac})_{3}$ to $10 \mathrm{~mol} \%$ or an increased amount of DTBP to 5.0 equiv and a lower temperature (110 ${ }^{\circ} \mathrm{C}$ ), decreased the yield to $63 \%, 69 \%$ and $79 \%$, respectively (Table 1, entries 12-14). The reaction did not proceed without the iron catalyst or DTBP (Table 1, entries 15 and 16).

We then examined the substrate scope and limitation of the procedure by reacting cyclohexane with a variety of substituted cinnamic acid derivatives under the optimized conditions (Table 1, entry 8). As shown in Scheme 1, almost all of the tested substrates worked well in this reaction. Several substituents on the aromatic ring were tolerated and the position of these substituents showed almost no effect on the chemical yield. We also observed that electron-donating substituents, such as methyl or methoxy groups at any position of the ring, efficiently took part in the reaction with a slightly decreased yield in case of ortho-substituted products (Scheme 1, 3b-g).

Table 1: Optimization of typical reaction conditions. ${ }^{a}$

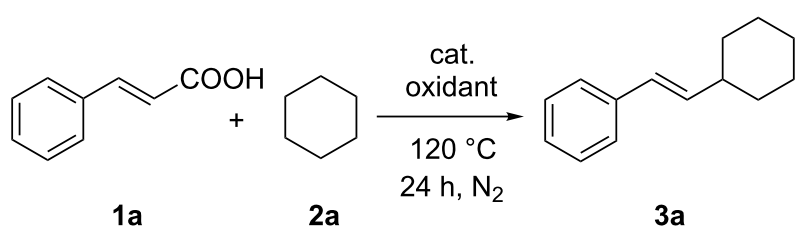

\begin{tabular}{|c|c|c|c|}
\hline Entry & cat. (mol \%) & oxidant & yield $(\%)^{b}$ \\
\hline 1 & $\mathrm{FeCl}_{2} \cdot 4 \mathrm{H}_{2} \mathrm{O}(20)$ & DTBP & 54 \\
\hline 2 & $\mathrm{FeCl}_{2} \cdot 4 \mathrm{H}_{2} \mathrm{O}(20)$ & TBHP & 38 \\
\hline 3 & $\mathrm{FeCl}_{2} \cdot 4 \mathrm{H}_{2} \mathrm{O}(20)$ & DTBP & $68^{c}$ \\
\hline 4 & $\mathrm{FeCl}_{3}(20)$ & DTBP & Trace \\
\hline 5 & Ferrocene (20) & DTBP & 74 \\
\hline 6 & $\mathrm{Fe}_{2} \mathrm{O}_{3}(20)$ & DTBP & 78 \\
\hline 7 & $\mathrm{Fe}_{3} \mathrm{O}_{4}(20)$ & DTBP & 80 \\
\hline 8 & $\mathrm{Fe}(\mathrm{acac})_{3}(20)$ & DTBP & 91 \\
\hline 9 & $\mathrm{Fe}(\mathrm{acac})_{3}(20)$ & $\mathrm{K}_{2} \mathrm{~S}_{2} \mathrm{O}_{8}$ & N.D. \\
\hline 10 & $\mathrm{Fe}(\mathrm{acac})_{3}(20)$ & $\mathrm{H}_{2} \mathrm{O}_{2}{ }^{\mathrm{d}}$ & 21 \\
\hline 11 & $\mathrm{Fe}(\mathrm{acac})_{3}(20)$ & TBPB & 49 \\
\hline 12 & $\mathrm{Fe}(\mathrm{acac})_{3}(10)$ & DTBPe & 63 \\
\hline $13^{f}$ & $\mathrm{Fe}(\mathrm{acac})_{3}(20)$ & DTBP & 69 \\
\hline 14 & $\mathrm{Fe}(\operatorname{acac})_{3}(20)^{\mathrm{g}}$ & DTBP & 79 \\
\hline 15 & $\mathrm{Fe}(\mathrm{acac})_{3}(20)$ & - & N.D. \\
\hline 16 & - & DTBP & N.D. \\
\hline
\end{tabular}

${ }^{a}$ Catalytic conditions: cinnamic acid $(0.3 \mathrm{mmol})$, cyclohexane $(2 \mathrm{~mL})$, iron catalyst $(20 \mathrm{~mol} \%)$, oxidant $\left(2.0\right.$ equiv), $120{ }^{\circ} \mathrm{C}, 24 \mathrm{~h}, \mathrm{~N}_{2} .{ }^{\mathrm{b}}$ Isolated yields based on cinnamic acid. 'Using 1,10-phenanthroline (30 mol \%) as the ligand. ${ }^{d} 30 \%$ aqueous solution. ${ }^{e} 5$ equiv. ${ }^{f} 12 \mathrm{~h}$. ${ }^{9} 110{ }^{\circ} \mathrm{C}$. 
Furthermore, a reaction of 2,6-disubstituted cinnamic acid $\mathbf{1 m}$ lead to the expected product $\mathbf{3 m}$, which was obtained in a lower yield due to steric hindrance. In addition, heteroaryl-substituted acrylic acids can also be efficiently converted under these conditions. This was shown by the reaction of 3-(thiophen-2yl)acrylic acid (1p) with cyclohexane furnishing the product 3p in $89 \%$ yield. In general, the stereoselectivity of this reaction was excellent and only trans-isomers were obtained in all cases.

Next, other cycloalkanes, including cyclopentane, cycloheptane and cyclooctane, were reacted with different cinnamic acids $\mathbf{1}$, giving products $\mathbf{4 a}-\mathbf{f}$ in $83-95 \%$ chemical yield (Scheme 2). As already shown for cyclohexane as a substrate, the reaction of other cycloalkanes performs equally well with a variety of cinammic acid derivatives under these conditions. It is noteworthy, that the decarboxylative cross-coupling with cyclooctane showed a higher efficiency than with smaller cycloalkanes.
Finally two control experiments were carried out to shed light on the reaction mechanism. Addition of the radical scavenger 2,2,6,6-tetramethylpiperidine $N$-oxide (TEMPO) or azobisisobutyronitrile (AIBN) completely inhibited the reaction, and almost no desired product was obtained. Based on these results and literature reports $[69,70]$, a plausible mechanism for the radical oxidative coupling is illustrated in Scheme 3. At the beginning, Fe-catalyzed cleavage of DTBP by Fe(III) in the presence of cinnamic acid, gives tert-butoxy radical $\mathbf{A}$, intermediate $\mathbf{B}$ and one acac. Next, a cyclohexane radical $\mathbf{C}$ is generated by the reaction between tert-butoxy radical $\mathbf{A}$ and cyclohexane. Subsequently, addition of cyclohexane radical $\mathbf{C}$ to the $\alpha$-position of the double bond in $\mathbf{B}$ gives intermediate $\mathbf{D}$. Finally, the radical intermediate $\mathbf{D}$ is oxidatively decarboxylated by $\mathrm{Fe}$ (III) to give product $3, \mathrm{Fe}(\mathrm{II})$ and carbon dioxide. The Fe(III) catalyst is then reformed via DTBP oxidation [71].

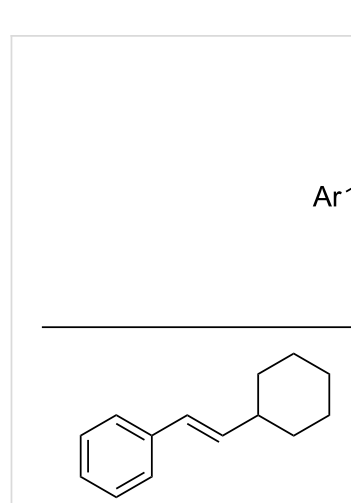

$3 a, 91 \%$<smiles>COc1ccc(/C=C/C2CCCCC2)cc1</smiles>

$3 e, 89 \%$<smiles>Fc1cccc(/C=C/C2CCCCC2)c1</smiles>

$3 \mathbf{i}, 88 \%$<smiles>Clc1cccc(Cl)c1/C=C/C1CCCCC1</smiles>

$3 \mathrm{~m}, 71 \%$<smiles>COc1cccc(/C=C/C2CCCCC2)c1</smiles>

3f, $92 \%$<smiles>Fc1ccccc1/C=C/C1CCCCC1</smiles>

3j, $80 \%$<smiles>Brc1ccc(/C=C/C2CCCCC2)cc1</smiles>

$3 n, 89 \%$
$\mathrm{Fe}(\mathrm{acac})_{3}(20 \mathrm{~mol} \%)$

DTBP ( 2 equiv)

$120^{\circ} \mathrm{C}, 24 \mathrm{~h}$<smiles>[Al]C=CC1CCCCC1</smiles>

$3 a-p$<smiles>Cc1ccccc1/C=C/C1CCCCC1</smiles>

$3 d, 84 \%$ 3c, $90 \%$<smiles>COc1ccccc1/C=C/C1CCCCC1</smiles>

$3 g, 85 \%$<smiles>Clc1ccc(/C=C/C2CCCCC2)cc1</smiles>

$3 k, 84 \%$<smiles>FC(F)(F)c1cccc(C=CC2CCCCC2)c1</smiles>

$30,84 \%$<smiles>Fc1ccc(/C=C/C2CCCCC2)cc1</smiles>

$3 h, 84 \%$<smiles>Clc1ccccc1/C=C/C1CCCCC1</smiles>

$31,82 \%$

Scheme 1: $\mathrm{Fe}(\mathrm{acac})_{3}$-catalyzed alkenylation of cyclohexane. Catalytic conditions: cinnamic acid (1) $(0.3 \mathrm{mmol}), \mathrm{cyclohexane}(2 \mathrm{~mL}), \mathrm{Fe}(\mathrm{acac})_{3}(20$ mol \%), DTBP (2.0 equiv), $120^{\circ} \mathrm{C}, 24 \mathrm{~h}, \mathrm{~N}_{2}$. Yields are isolated yields based on cinnamic acid. 


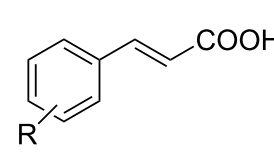

1

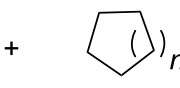

2b-d

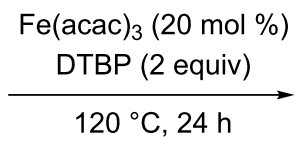

$120^{\circ} \mathrm{C}, 24 \mathrm{~h}$<smiles>[R]c1cccc(/C=C/C2CCCC2)c1</smiles>

4a-f<smiles>C(=C/C1CCCC1)\c1ccccc1</smiles>

4a, $92 \%$<smiles>Clc1ccccc1/C=C/C1CCCC1</smiles>

4d, $83 \%$<smiles>Cc1ccc(/C=C/C2CCCC2)cc1</smiles>

4b, $90 \%$<smiles>C(=C/C1CCCCCC1)\c1ccccc1</smiles>

$4 e, 89 \%$<smiles>Fc1ccc(/C=C/C2CCCC2)cc1</smiles>

4c, $87 \%$<smiles>C(=C/C1CCCCCCC1)\c1ccccc1</smiles>

4f, $95 \%$

Scheme 2: $\mathrm{Fe}(\mathrm{acac})_{3}$-catalyzed alkenylation of cyclopentan, cycloheptane and cyclooctane. Catalytic conditions: cinnamic acid (1) (0.3 mmol), cycloalkanes $(2.0 \mathrm{~mL}), \mathrm{Fe}(\mathrm{acac})_{3}(20 \mathrm{~mol} \%)$, DTBP $\left(2\right.$ equiv), $120^{\circ} \mathrm{C}, 24 \mathrm{~h}, \mathrm{~N}_{2}$. Yields are isolated yields based on cinnamic acid.
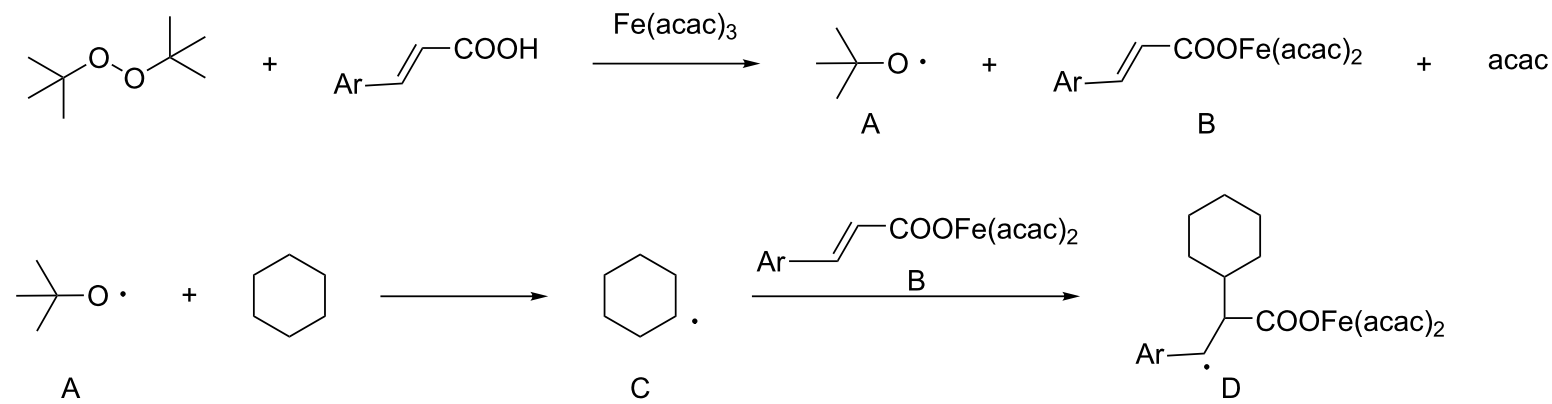<smiles>CC(CBr)COC(=O)C1CCCCC1</smiles>

D
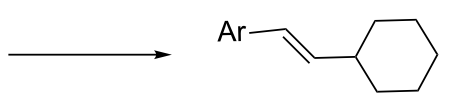

$+\mathrm{Fe}(\mathrm{acac})_{2}+\mathrm{CO}_{2}$<smiles>CCCCCCCCO</smiles>

3

Scheme 3: A plausible pathway for the reaction.

\section{Conclusion}

In conclusion, an efficient procedure for the $\mathrm{Fe}(\mathrm{acac})_{3}$-catalyzed direct alkenylation of $\mathrm{sp}^{3} \mathrm{C}-\mathrm{H}$ bonds of cycloalkanes with DTBP as an oxidant has been reported. This method provides a useful strategy for the stereospecific synthesis of substituted E-alkenes. Various cinnamic acids and cycloalkanes are welltolerated in this catalytic system with good to excellent chemical yields. The mechanism of this reaction has also been 
studied, and a radical mechanism was proposed. Further studies on the alkenylation of other $\mathrm{sp}^{3} \mathrm{C}-\mathrm{H}$ substrates are currently investigated in our laboratory.

\section{Experimental}

General procedure for the iron-catalyzed decarboxylative alkenylation of cycloalkanes: To a Schlenk tube equipped with a magnetic stir bar were added $\mathrm{Fe}(\mathrm{acac})_{3}(21.2 \mathrm{mg}, 0.06 \mathrm{mmol})$ and cinnamic acid $(0.3 \mathrm{mmol})$ under a nitrogen atmosphere. Cycloalkane $(2.0 \mathrm{~mL}, 15-25 \mathrm{mmol})$ and DTBP (di-tert-butyl peroxide, $0.6 \mathrm{mmol}, 113 \mu \mathrm{L}$ ) were added under a nitrogen atmosphere and the resulting reaction mixture was stirred at 120 ${ }^{\circ} \mathrm{C}$ for $24 \mathrm{~h}$. After cooling to room temperature and removal of volatiles, the products were isolated by flash column chromatography (PE).

\section{Supporting Information}

\section{Supporting Information File 1}

Experimental details and spectral data.

[http://www.beilstein-journals.org/bjoc/content/

supplementary/1860-5397-9-197-S1.pdf]

\section{Acknowledgements}

We gratefully acknowledge the financial support from the National Natural Science Foundation of China (No. 21102071) and the Fundamental Research Funds for the Central Universities (No. 1107020522 and No. 1082020502). The Jiangsu 333 program (for Pan) and Changzhou Jin-Feng-Huang program (for Han) are also acknowledged.

\section{References}

1. Chen, X.; Engle, K. M.; Wang, D.-H.; Yu, J.-Q. Angew. Chem., Int. Ed. 2009, 48, 5094-5115. doi:10.1002/anie.200806273

2. Daugulis, O.; Do, H.-Q.; Shabashov, D. Acc. Chem. Res. 2009, 42, 1074-1086. doi:10.1021/ar9000058

3. Li, C.-J. Acc. Chem. Res. 2009, 42, 335-344. doi:10.1021/ar800164n

4. Coperet, C. Chem. Rev. 2010, 110, 656-680. doi:10.1021/cr900122p

5. Werner, H. Angew. Chem., Int. Ed. 2010, 49, 4714-4728. doi:10.1002/anie.201000306

6. Sun, C.-L.; Li, B.-J.; Shi, Z.-J. Chem. Rev. 2011, 111, 1293-1314. doi:10.1021/cr100198w

7. Campbell, A. N.; Stahl, S. S. Acc. Chem. Res. 2012, 45, 851-863. doi:10.1021/ar2002045

8. Kozhushkov, S. I.; Ackermann, L. Chem. Sci. 2013, 4, 886-896. doi:10.1039/c2sc21524a

9. Chatani, N.; Asaumi, T.; Yorimitsu, S.; Ikeda, T.; Kakiuchi, F.; Murai, S. J. Am. Chem. Soc. 2001, 123, 10935-10941. doi:10.1021/ja011540e

10. Jiang, X. F.; Shen, M.; Tang, Y.; Li, C. Tetrahedron Lett. 2005, 46, 487-489. doi:10.1016/j.tetlet.2004.11.113

11. Song, C.-X.; Cai, G.-X.; Farrell, T. R.; Jiang, Z.-P.; Li, H.; Gan, L.-B.; Shi, Z.-J. Chem. Commun. 2009, 6002-6004. doi:10.1039/b911031c
12. Guin, S.; Rout, S. K.; Banerjee, A.; Nandi, S.; Patel, B. K. Org. Lett. 2012, 14, 5294-5297. doi:10.1021/ol302438z

13. Rousseaux, S.; Liégault, B.; Fagnou, K. Chem. Sci. 2012, 3, 244-248. doi:10.1039/c1sc00458a

14. Solé, D.; Mariani, F.; Fernández, I.; Sierra, M. A. J. Org. Chem. 2012, 77, 10272-10284. doi:10.1021/jo301924e

15. Wasa, M.; Chan, K. S. L.; Zhang, X.-G.; He, J.; Miura, M.; Yu, J.-Q. J. Am. Chem. Soc. 2012, 134, 18570-18572. doi:10.1021/ja309325e

16. Yin, Z.; Sun, P. J. Org. Chem. 2012, 77, 11339-11344. doi:10.1021/jo302125h

17. Saget, T.; Perez, D.; Cramer, N. Org. Lett. 2013, 15, 1354-1357. doi:10.1021/ol400380y

18. Zhang, S.-Y.; He, G.; Nack, W. A.; Zhao, Y.; Li, Q.; Chen, G. J. Am. Chem. Soc. 2013, 135, 2124-2127. doi:10.1021/ja312277g

19. Li, Z.; Li, C.-J. J. Am. Chem. Soc. 2004, 126, 11810-11811. doi:10.1021/ja0460763

20. Xie, J.; Huang, Z.-Z. Angew. Chem., Int. Ed. 2010, 49, 10181-10185. doi:10.1002/anie.201004940

21. Yang, F.; Li, J.; Xie, J.; Huang, Z.-Z. Org. Lett. 2010, 12, 5214-5217. doi:10.1021/ol102252n

22. Rout, S. K.; Guin, S.; Ghara, K. K.; Banerjee, A.; Patel, B. K. Org. Lett. 2012, 14, 3982-3985. doi:10.1021/ol301756y

23. Xia, R.; Niu, H.-Y.; Qu, G.-R.; Guo, H.-M. Org. Lett. 2012, 14, 5546-5549. doi:10.1021/ol302640e

24. Pastine, S. J.; Gribkov, D. V.; Sames, D. J. Am. Chem. Soc. 2006, 128, 14220-14221. doi:10.1021/ja064481j

25. Rankin, M. A.; Schatte, G.; McDonald, R.; Stradiotto, M. J. Am. Chem. Soc. 2007, 129, 6390-6391. doi:10.1021/ja071684e

26. Deng, G.; Zhao, L.; Li, C.-J. Angew. Chem., Int. Ed. 2008, 47, 6278-6282. doi:10.1002/anie.200801544

27. Wang, M.-Z.; Zhou, C.-Y.; Wong, M.-K.; Che, C.-M. Chem.-Eur. J. 2010, 16, 5723-5735. doi:10.1002/chem.200902387

28. Shi, L.; Tu, Y.-Q.; Wang, M.; Zhang, F.-M.; Fan, C.-A.; Zhao, Y.-M.; Xia, W.-J. J. Am. Chem. Soc. 2005, 127, 10836-10837. doi:10.1021/ja0528331

29. Jo, E.-A.; Lee, J.-H.; Jun, C.-H. Chem. Commun. 2008, 5779-5781. doi:10.1039/b814166e

30. Rakshit, S.; Patureau, F. W.; Glorius, F. J. Am. Chem. Soc. 2010, 132, 9585-9587. doi:10.1021/ja104305s

31. Kuninobu, Y.; Nakahara, T.; Takeshima, H.; Takai, K. Org. Lett. 2013, 15, 426-428. doi:10.1021/ol303353m

32. Harden, J. D.; Ruppel, J. V.; Gao, G.-Y.; Zhang, X. P. Chem. Commun. 2007, 4644-4646. doi:10.1039/b710677g

33. Hung-Low, F.; Krogman, J. P.; Tye, J. W.; Bradley, C. A. Chem. Commun. 2012, 48, 368-370. doi:10.1039/c1cc15458c

34. Lu, H.; Hu, Y.; Jiang, H.; Wojtas, L.; Zhang, X. P. Org. Lett. 2012, 14, 5158-5161. doi:10.1021/ol302511f

35. Horino, Y.; Yamamoto, T.; Ueda, K.; Kuroda, S.; Toste, F. D. J. Am. Chem. Soc. 2009, 131, 2809-2811. doi:10.1021/ja808780r

36. Bhunia, S.; Ghorpade, S.; Huple, D. B.; Liu, R.-S. Angew. Chem., Int. Ed. 2012, 51, 2939-2942. doi:10.1002/anie.201108027

37. DeBoef, B.; Pastine, S. J.; Sames, D. J. Am. Chem. Soc. 2004, 126, 6556-6557. doi:10.1021/ja049111e

38. Pan, S.; Endo, K.; Shibata, T. Org. Lett. 2011, 13, 4692-4695. doi:10.1021/ol201907w

39. Obora, Y.; Ogawa, S.; Yamamoto, N. J. Org. Chem. 2012, 77, 9429-9433. doi:10.1021/jo3019347

40. Wang, Z.; Zhang, Y.; Fu, H.; Jiang, Y.; Zhao, Y. Org. Lett. 2008, 10 , 1863-1866. doi:10.1021/ol800593p 
41. Zhang, S.-Y.; Tu, Y.-Q.; Fan, C.-A.; Zhang, F.-M.; Shi, L. Angew. Chem., Int. Ed. 2009, 48, 8761-8765. doi:10.1002/anie.200903960

42. Pan, S.; Liu, J.; Li, H.; Wang, Z.; Guo, X.; Li, Z. Org. Lett. 2010, 12, 1932-1935. doi:10.1021/ol100670m

43. Bloom, S.; Pitts, C. R.; Woltornist, R.; Griswold, A.; Holl, M. G.; Lectka, T. Org. Lett. 2013, 15, 1722-1724. doi:10.1021/ol400424s

44. Vadola, P. A.; Sames, D. J. Am. Chem. Soc. 2009, 131, 16525-16528. doi:10.1021/ja906480w

45. Yoshikai, N.; Mieczkowski, A.; Matsumoto, A.; Ilies, L.; Nakamura, E. J. Am. Chem. Soc. 2010, 132, 5568-5569. doi:10.1021/ja100651t

46. Qian, B.; Xie, P.; Xie, Y.; Huang, H. Org. Lett. 2011, 13, 2580-2583. doi:10.1021/ol200684b

47. Liu, X.; Sun, B.; Xie, Z.; Qin, X.; Liu, L.; Lou, H. J. Org. Chem. 2013, 78, 3104-3112. doi:10.1021/j04000674

48. Chen, L.; Shi, E.; Liu, Z.; Chen, S.; Wei, W.; Li, H.; Xu, K.; Wan, X. Chem.-Eur. J. 2011, 17, 4085-4089. doi:10.1002/chem.201100192

49. He, T.; Yu, L.; Zhang, L.; Wang, L.; Wang, M. Org. Lett. 2011, 13, 5016-5019. doi:10.1021/ol201779n

50. Huang, J.; Li, L.-T.; Li, H.-Y.; Husan, E.; Wang, P.; Wang, B. Chem. Commun. 2012, 48, 10204-10206. doi:10.1039/c2cc35450k

51. Li, L.-T.; Li, H.-Y.; Xing, L.-J.; Wen, L.-J.; Wang, P.; Wang, B. Org. Biomol. Chem. 2012, 10, 9519-9522. doi:10.1039/c2ob26636a

52. Mai, W.-P.; Wang, H.-H.; Li, Z.-C.; Yuan, J.-W.; Xiao, Y.-M.; Yang, L.-R.; Mao, P.; Qu, L.-B. Chem. Commun. 2012, 48, 10117-10119. doi:10.1039/c2cc35279f

53. Feng, J.; Liang, S.; Chen, S.-Y.; Zhang, J.; Fu, S.-S.; Yu, X.-Q. Adv. Synth. Catal. 2012, 354, 1287-1292. doi:10.1002/adsc.201100920

54. Shi, E.; Shao, Y.; Chen, S.; Hu, H.; Liu, Z.; Zhang, J.; Wan, X. Org. Lett. 2012, 14, 3384-3387. doi:10.1021/ol3013606

55. Wang, F.-F.; Luo, C.-P.; Wang, Y.; Deng, G.; Yang, L. Org. Biomol. Chem. 2012, 10, 8605-8608. doi:10.1039/c2ob26604k

56. Zhu, Y.-p.; Jia, F.-c.; Liu, M.-c.; Wu, L.-m.; Cai, Q.; Gao, Y.; Wu, A.-X. Org. Lett. 2012, 14, 5378-5381. doi:10.1021/ol302613q

57. Zhu, Y.-p.; Liu, M.-c.; Jia, F.-c.; Yuan, J.-j.; Gao, Q.-h.; Lian, M.; Wu, A.-x. Org. Lett. 2012, 14, 3392-3395. doi:10.1021/ol301366p

58. Xiang, J.; Fuchs, P. L. J. Am. Chem. Soc. 1996, 118, 11986-11987. doi:10.1021/ja962790b

59. Jang, Y.-J.; Shih, Y.-K.; Liu, J.-Y.; Kuo, W.-Y.; Yao, C.-F. Chem.-Eur. J. 2003, 9, 2123-2128. doi:10.1002/chem.200204571

60. Cui, Z.; Shang, X.; Shao, X.-F.; Liu, Z.-Q. Chem. Sci. 2012, 3, 2853-2858. doi:10.1039/c2sc20712e

61. Forgione, P.; Brochu, M.-C.; St-Onge, M.; Thesen, K. H.; Bailey, M. D.; Bilodeau, F. J. Am. Chem. Soc. 2006, 128, 11350-11351. doi:10.1021/ja063511f

62. Gooßen, L. J.; Rudolphi, F.; Oppel, C.; Rodríguez, N. Angew. Chem., Int. Ed. 2008, 47, 3043-3045. doi:10.1002/anie.200705127

63. Kim, H.; Lee, P. H. Adv. Synth. Catal. 2009, 351, 2827-2832. doi:10.1002/adsc.200900502

64. Wang, Z.; Ding, Q.; He, X.; Wu, J. Org. Biomol. Chem. 2009, 7, 863-865. doi:10.1039/b821870f

65. Zhang, F.; Greaney, M. F. Angew. Chem., Int. Ed. 2010, 49, 2768-2771. doi:10.1002/anie.200906921

66. Wang, Z. T.; Zhu, L.; Yin, F.; Su, Z. Q.; Li, Z.; Li, C. J. Am. Chem. Soc. 2012, 134, 4258-4263. doi:10.1021/ja210361z

67. Yin, F.; Wang, Z.; Li, Z.; Li, C. J. Am. Chem. Soc. 2012, 134, 10401-10404. doi:10.1021/ja3048255
68. Liu, X.; Wang, Z.; Cheng, X.; Li, C. J. Am. Chem. Soc. 2012, 134, 14330-14333. doi:10.1021/ja306638s

69. Liu, Z.-Q.; Sun, L.; Wang, J.-G.; Han, J.; Zhao, Y.-K.; Zhou, B. Org. Lett. 2009, 11, 1437-1439. doi:10.1021/ol900145u

70. Yang, H.; Yan, H.; Sun, P.; Zhu, Y.; Lu, L.; Liu, D.; Rong, G.; Mao, J. Green Chem. 2013, 15, 976-981. doi:10.1039/c3gc37131j

71. Chowdhury, S.; Roy, S. J. Org. Chem. 1997, 62, 199-200. doi:10.1021/jo951991f

\section{License and Terms}

This is an Open Access article under the terms of the Creative Commons Attribution License

(http://creativecommons.org/licenses/by/2.0), which permits unrestricted use, distribution, and reproduction in any medium, provided the original work is properly cited.

The license is subject to the Beilstein Journal of Organic Chemistry terms and conditions:

(http://www.beilstein-journals.org/bjoc)

The definitive version of this article is the electronic one which can be found at: doi:10.3762/bjoc.9.197 